\title{
Is Minimally Invasive Mitral Valve Surgery
}

Inappropriate for Redo-surgery or Elderly Patients?

- Study of 55 Consecutive Cases Following

Minimally Invasive Mitral Valve Replacement Via

Right Mini-thoracotomy

\section{Taisuke Nakayama}

Chiba-Nishi general hospital

Yoshitsugu Nakamura ( $\nabla$ ystgnkmr@gmail.com )

Department of Cardiovascular Surgery, Chiba-Nishi General Hospital, Chiba, Japan 107-1 Kanegasaku Matsudo-shi, Chiba-Ken 270-2251, Japan

\section{Yuto Yasumoto}

Chiba-Nishi general hospital

\section{Daiki Yoshiyama}

Chiba-Nishi general hospital

Miho Kuroda

Chiba-Nishi general hospital

Shuhei Nishijima

Chiba-Nishi general hospital

\section{Ryo Tsuruta}

Chiba-Nishi general hospital

\section{Takuya Narita}

Chiba-Nishi general hospital

\section{Yujiro Ito}

Chiba-Nishi general hospital

\section{Research article}

Keywords: minimally invasive surgery, mitral valve replacement, right mini-thoracotomy, redo-surgery, elderly

Posted Date: July 2nd, 2021

DOl: https://doi.org/10.21203/rs.3.rs-667064/v1 
License: (c) (i) This work is licensed under a Creative Commons Attribution 4.0 International License. Read Full License 


\section{Abstract}

Background: Minimally invasive mitral valve surgery has been established as a routine procedure at our institution. In the present study, early and mid-term outcomes of patients who underwent minimally invasive mitral valve replacement (MIMVR), including redo-operations and elderly patients, during the 5year post-surgery period were analyzed to review short-term morbidity and mortality, and mid-term results.

Methods: Preoperative variables, intraoperative findings, and postoperative outcomes of MIMVR patients treated from January 2014 to November 2020 and prospectively stored in a database were reviewed. Survival and freedom from cerebrovascular events were evaluated using life tables and Kaplan-Meier analysis.

Results: A total of 445 patients underwent minimally invasive mitral valve surgery during the study period, of whom 55 received mitral valve replacement (MVR), including 18 cases of redo-MVR and 10 elderly ( $\geq 80$ years old) patients. Mean age at the time of surgery was $70.7 \pm 11.3$ years. The number of patients who underwent conversion to a sternotomy was 0 , while 30 -day mortality was noted in one (2\%). For all MIMVR cases, 1- and 5-year survival was $90.8 \pm 3.9 \%$ and $76.5 \pm 7.1 \%$, respectively. Furthermore, freedom from cerebrovascular events and anticoagulation-related complications was $94.3 \pm 3.2 \%$ and $84.2 \pm 6.3 \%$ at 1 and 5 years, respectively. In univariate analysis, independent predictors of hospital mortality and prolonged hospital stay included infectious endocarditis, while previous cardiac surgery and elderly status were not significant factors.

Conclusions: MIMVR can be performed safely and effectively for redo-MVR and in elderly patients with very few perioperative complications. Early and mid-term outcomes in the present cohort were acceptable.

\section{Introduction}

The feasibility and safety of minimally invasive mitral valve surgery (MIMVS) with the primary goals of improved cosmetic factors and reduced postoperative discomfort, while maintaining the same level of safety and efficacy as with conventional surgery, have been demonstrated in several reports [1]. Most analyses have shown that patients who undergo MIMVS are at low risk and young, and have fewer comorbidities [1]. However, questions remain regarding whether these methods should be limited to young patients or primary cases. On the other hand, few studies have specifically investigated minimally invasive mitral valve replacement (MIMVR) in detail.

Although catheter use is extremely common, a recent search of the Society of Thoracic Surgeons database showed that the number of mitral valve operation procedures (valve replacement or valvuloplasty) during the period from 2014 through 2019 was not decreased. For patients who underwent isolated mitral valve replacement (MVR), the operative and hospital death rates were $4.6 \%$ and $4.0 \%$, respectively, which were higher as compared to those for cases of isolated aortic valve replacement $(A V R)$, coronary artery bypass grafting (CABG), or mitral valve(MV) repair. Furthermore, the major 
morbidity rate (21.7\%) was also elevated [2]. It is anticipated that results will improve with use of an MIMVS procedure.

A mitral valve re-operation can be demanding for patients with a patent coronary artery bypass graft or who have undergone a previous aortic valve replacement procedure, as well as those affected by complications following a previous operation (abscess, perivalvular leakage, thrombosis). In addition, risk of graft injury or hemorrhage, presence of dense adhesions, and/or complex valve exposure can make a redo-valve operation through a median sternotomy challenging [3]. In such cases, a minimally invasive surgical approach through a right-sided mini-thoracotomy is considered to be a valid alternative instead of a repeated conventional median sternotomy [4].

In 2014, use of an MIVMR procedure was established at our department, with that via a right minithoracotomy (RT approach) now the standard used for treatment of mitral valve disease. The aim of the present study was to analyze early and mid-term outcomes of 55 consecutive patients who underwent MIMVR via an RT approach during a recent 5-year period. In addition, to determine the effectiveness of redo-MIMVR and the procedure in elderly ( $\geq 80$ years old) patients, we compared the results of primary and redo-MIMVR cases, and also examined adaptation for elderly cases.

\section{Methods}

The study complies with the Declaration of Helsinki. The present analysis was approved by the audit board at the Chiba-Nishi general hospital. As this analysis came under clinical audit/quality of care assessment and all data were anonymized following the governance criteria of the NHS, the IRB agreed informed consent was not required.

\section{Patient selection and data collection}

A total of 445 patients underwent minimally invasive mitral valve surgery via an RT approach at ChibaNishi General Hospital between January 2014 and November 2020. Of the patients treated during the study period, 380 underwent mitral valve repair as the primary procedure, while 65 underwent MIMVR via an RT approach. After excluding 10 of the MIMVR patients who received concomitant aortic valve replacement, 55, including 10 classified as elderly, who underwent that as first-time $(n=38)$ or redo $(n=17)$ procedure were enrolled, each of whom also had late follow-up results available.

The main outcomes investigated were early and late mortality, perioperative complications, and freedom from cerebrovascular events and reoperation. Early mortality was defined as death from any cause occurring within 30 days of the operation or before hospital discharge. Stroke was defined as any new focal or global neurological deficit lasting more than 24 hours,.

All patients were examined postoperatively from 1 to 4 weeks, and again at 12 and 24 weeks, then were contacted thereafter for follow-up information. Follow-up examinations were performed every 24 weeks in 
the late phase. The median follow-up period was $34.2 \pm 24.6$ months (interquartile range $13-52$ months) and follow-up examinations were performed in $100 \%$ of the cases.

\section{Statistical analysis}

Continuous data are expressed as the mean \pm standard deviation or median with interquartile range (IQR), while categorical data are shown as percentages. Survival and time-to-event analyses for rates of cerebrovascular events and anticoagulation-related complications were assessed using the Kaplan-Meier method. All reported $P$ values are two-sided and those $<0.05$ were considered to indicate statistical significance. All statistical analyses of recorded data were performed using the Excel statistical software package (Ekuseru-Toukei 2010; Social Survey Research Information Co., Ltd., Tokyo, Japan).

\section{Results}

Baseline characteristics and risk factor prevalence are listed in Table 1. The mean age of the enrolled patients was $70.7 \pm 11.3$ years and $36(65 \%)$ were female. Eighteen (33\%) had previously undergone a cardiac operation, including MV repair in $8, M V R$ in 1, AVR in 3, total arch replacement in 2, and CABG in 1. The mean preoperative left ventricular ejection fraction was $60.6 \pm 11.7 \%$. Preoperative chronic atrial fibrillation was noted in 20 (36\%) patients. There were no statistically significant differences between first-time and redo-MIMVR patients for mean age $(71.8 \pm 10.1$ vs. $68.4 \pm 14.6 \%, p=0.31)$. The redo population had a significantly higher prevalence for EuroSCORE (4.37 \pm 4.7 vs. $7.79 \pm 6.2, p=0.03)$ and left ventricular ejection fraction dysfunction $(62.8 \pm 11.5$ vs. $56.2 \pm 11.5 \%, p=0.05)$, whereas there was no statistically significant difference between the elderly and younger (age $<80$ years) patients for EuroSCORE (7.95 \pm 6.7 vs. $4.94 \pm 5.0, p=0.11)$. On the other hand, the elderly group had a significantly higher prevalence for diabetes (insulin user) [4 (40\%) vs. $6(13 \%), p=0.048]$ and pulmonary hypertension (>45 mmHg) [7 (70\%) vs. $16(36), p=0.046]$. The most predominant pathological factor was rheumatic disease $(n=29,52 \%)$, followed by degenerative disease $(n=10,18 \%)$ and endocarditis $(n=8,15 \%)$, with other such factors noted in 8 (15\%).

The operative procedural details are presented in Table 2. All 55 patients underwent a successful MIMVR via an RT. Seven received a mechanical valve and 48 a bioprosthetic valve, and all had an uneventful recovery. There were no cases of intraoperative conversion to a sternotomy. The mean operative time was $215 \pm 46.7$ minutes, while aortic cross-clamp and cardiopulmonary (CPB) times were $98 \pm 26.8$ and $131 \pm 28.6$ minutes, respectively, for the MIMVR. There were no statistically significant differences between first-time and redo-MIMVR patients in regard to CPB $(129 \pm 28.1$ vs. $137 \pm 30.7$ minutes, $p=0.36)$ or aortic cross-clamp ( $100 \pm 24.4$ vs. $96 \pm 32.4$ minutes, $p=0.62)$ times, though redo-MIMVR patients had a longer operation time $(207 \pm 46.5$ vs. $233 \pm 44.2$ minutes, $p=0.046)$. There was no statistically significant difference between the elderly and younger patients for operation time. Concomitant procedures were performed in 24 patients (44\%), including atrial fibrillation (AF) ablation in 17 (31\%), tricuspid valve surgery in $11(20 \%)$, patent foramen ovale/atrial septal defect closure in $2(4 \%)$, and oversewing or use of 
a closed devise for the left atrial appendage in 15 (27\%). Redo-MIMVR patients were less likely to undergo a concomitant procedure [21 (57\%) vs. $3(17 \%), p=0.005]$.

\section{Early outcomes}

Operative results are presented in Table 3. There was 1 in-hospital mortality (2\%), which occurred on postoperative day 92 and was due to acute respiratory distress syndrome in an active IE patient. Median hospital and ICU stays were 11 (IQR 8.8-18) and 3 (IQR 2-4) days, respectively. There was no statistically significant difference between elderly and younger patients in regard to intensive care unit (ICU) length of stay [3 (2.3-4) vs. $3(2-4)$ days, $p=0.61$ ], though the elderly group had longer hospital length of stay [16.5 (13-33) vs. 10 (8-15.8) days, $p=0.01]$ and ventilation time [16 (12.5-18) vs. 8 (6-12) hours, $p=0.003]$. There were no statistically significant differences between first-time and redo-MIMVR cases in regard to ICU or hospital stay duration. A blood transfusion during hospitalization was required in 27 (49\%) patients, with those who underwent redo-MIMVR [14 (38\%) vs. $13(72 \%), p=0.017]$ or with elderly status [8 (80\%) vs. 19 $(42 \%), p=0.03$ ] more likely to undergo a transfusion. Of 35 patients admitted for sinus rhythm, new-onset atrial fibrillation occurred in $7(13 \%)$. No patients had a cerebral vascular accident, while $2(4 \%)$ had an acute renal failure incident that required dialysis, of whom $1(2 \%)$ needed a reoperation for bleeding. Following surgery, all patients underwent a transthoracic echocardiography examination prior to discharge, with no device-related complications or paravalvular leakage observed.

\section{Midterm outcomes}

Clinical follow-up examinations were performed in 55 patients (100\%) over a mean period of $34.2 \pm 24.6$ months (interquartile range 13-52 months). None demonstrated prosthetic valve dysfunction or underwent a mitral prosthetic valve reoperation.

\section{Survival}

Overall, the 1-, 3-, and 5-year survival rates were $90.8 \pm 3.9 \%, 81.3 \pm 5.9 \%$, and $76.5 \pm 7.1 \%$, respectively, while those for the elderly group were $80.0 \pm 12.7 \%, 64.0 \pm 17.5 \%$, and $64.0 \pm 17.5 \%$, respectively, and for the younger group were $93.3 \pm 3.8 \%, 84.3 \pm 6.1 \%$, and $78.6 \pm 7.8 \%$, respectively (Fig. 1A, B). A total of 10 patients (18\%), $3(30 \%)$ elderly and 7 (16\%) younger, died during the follow-up period, of whom $4(10.8 \%)$ underwent first-time MIMVR and 6 (33\%) redo-MIMVR. The cause of death could only be determined in 8 , which was cardiac-related in $3(3,14,30$ months), pneumonia in 2 ( 3,5 months), a cerebral vascular event in 1 (48 months), an abdominal aortic aneurysm rupture in 1 (19 months), septic shock in 1 (2 months), and unknown in 2 ( 3,13 months).

\section{Cerebrovascular events and anticoagulation-related complications}

Following mitral valve replacement, the rates for freedom from cerebrovascular events and anticoagulation-related complications after 1,3 , and 5 years were $94.3 \pm 3.2 \%, 88.4 \pm 5.1 \%$, and $84.2 \pm 6.3 \%$, respectively, while those in elderly patients were $100 \%, 100 \%$, and $100 \%$, respectively, and in the younger group were $93.2 \pm 3.8 \%, 86.5 \pm 5.8 \%$, and $81.7 \pm 7.2 \%$, respectively (Fig. $2 A, B$ ). 


\section{Predictors of hospital mortality and prolonged hospital stay shown by univariate analysis}

Following MIMVR surgery, there was 1 in-hospital mortality (2\%) and 7 patients had a prolonged ( $>30$ days) hospital stay $(12.7 \%)$, for a total of $8(14.5 \%)$ cases. Variables assessed as possible predictors of mortality in univariate analysis are shown in Table 4 . The only significant $(p=0.05)$ univariate predictor was infectious endocarditis, while previous cardiac surgery, concomitant operation, and elderly status were not found to be significant.

\section{Discussion}

Although several reports have described excellent early and long-term results of minimally invasive mitral valve repair [5], few studies have analyzed MIMVR cases in detail. The present analysis of a consecutive series of patients who underwent an MIMVR procedure found it to be safe and associated with excellent postoperative outcomes, while good procedural times, shorter hospital and ICU stays, and acceptable mid-term results were also revealed. As demonstrated by complete echocardiographic follow-up results, no paravalvular leakage developed in any of the patients, including during the late phase. Specifically, overall in-hospital mortality and postoperative neurological events were nearly $0 \%$, with the latter lower than the range of rates for mortality and neurological events recently presented by Modi and colleagues [6] in a meta-analysis of MIMVR cases (4.9\% in 5 cohorts with a total of 979 patients, $2.3 \%$ in 3 cohorts with a total of 778 patients).

In the present study, since there were no cerebral vascular accident incidents, preoperative contrast CT results were analyzed to detect aorta condition, i.e., the presence of arteriosclerotic disease or parietal thrombus, and determine the perfusion program for each case. Details of the perfusion protocol were previously reported by Nakamura et al. [7]. When significant arteriosclerotic disease was found in the entire aorta and iliac artery, right axillary artery cannulation was employed to establish antegrade perfusion for CPB.

Although early good results and quick recovery following MIMVR have been noted in prior reports [8], there are concerns regarding its long-term efficacy, which has not been adequately elucidated. We found 2 reports that included long-term follow-up findings after MIMVR procedures performed at high-volume centers. The longest functional and echocardiographic follow-up study was conducted by Glauber and colleagues, who analyzed 476 patients who underwent MIMVR via an RT approach between 2003 and 2013 (mean age $67 \pm 11$ years, incision length $5-7 \mathrm{~cm}$, in-hospital mortality $3.2 \%$, neurological complications $2.6 \%$, reoperation for bleeding $6.4 \%$ ) [9]. In those cases, survival after replacement at 1,5 , and 10 years was found to be $91.0 \pm 1.4 \%, 81.3 \pm 2.5 \%$, and $76.2 \pm 3.4 \%$ respectively, while the rates for freedom from reoperation after replacement at 1,5 , and 10 years were $98.6 \pm 0.6 \%, 94.5 \pm 1.6 \%$, and $83.9 \pm 5.5 \%$, respectively. In 2003 , Casselman et al. reported early and long-term results in a review of a series of 80 patients who underwent endoscopic MVR with an RT procedure (incision length $4 \mathrm{~cm}$, mean follow-up period $19.6 \pm 17.3$ months, completed in all) [10]. Although $2(2.5 \%)$ of their patients experienced new onset endocarditis during the follow-up period and 1 underwent a reoperation with a median 
sternotomy, they noted excellent freedom from late phase death (4 years) in $92.0 \pm 3.6 \%$ as well as freedom from anticoagulation-related complications (4 years) in $97.0 \pm 1.9 \%$. In the present cohort who underwent MIMVR (mean age $70.7 \pm 11.3$ years, mean incision length $5-6 \mathrm{~cm}$ ), 5-year survival, freedom from a mitral valve-related reoperation, and freedom from cerebrovascular events and anticoagulationrelated complications showed favorable rates of $76.5 \%, 100 \%$, and $81.7 \%$, respectively. In addition, those rates were also good after first-time MIMVR at $83 \%, 100 \%$, and $93 \%$, respectively. As compared to those two other recent reports, our mid-term survival results were relatively better, while the freedom from reoperation was $0 \%$ at the 5 -year follow-up examination, and there were no device-related complications or cases of paravalvular leakage.

Reoperative mitral valve surgery through a median sternotomy can be particularly challenging due to dense adhesions and is known to carry a substantial risk of injury to vascular structures, which have been found to occur in 7-9\% of cases and are associated with increased mortality [11]. A valid alternative that could be employed to avoid risks associated with a redo median sternotomy approach is a right anterolateral mini-thoracotomy approach [12]. Unfortunately, only 5 patients who underwent a conventional sternotomy were available as a control group for the present study. Our strategy consists of selecting MIMVR whenever possible even for redo-mitral valve procedure cases, as that procedure has been our the first-line choice since 2014. However, that introduces a critical selection bias including cases of redo-mitral valve surgery between MIMVR and conventional sternotomy groups for analysis of procedures performed at our institution. Thus, we sought to compare the present results with those previously reported for cases of redo-MIMVR or conventional full sternotomy redo-mitral valve surgery. Studies regarding the feasibility of redo-MIMVR via an RT procedure have been presented by Ricci et al. [13], Sharony et al. [14], and Thomson et al. [15], with excellent results reported in each (Table 5). The results obtained in the present cases are favorable as compared with those, though remaining issues related to our method include longer prolonged CPB and cross-clamp times as compared to those prior findings. On the other hand, there was only one in-hospital mortality among the present cohort. Furthermore, the rates for freedom from stroke events and reoperation for bleeding were nearly $0 \%$. In addition, 3 other retrospective observational studies all demonstrated the superiority of an RT approach as compared to a median sternotomy for reoperative mitral valve surgery, with excellent results noted in those cases (Table 5) [16-18]. Pooled analysis showed a mini-thoracotomy mitral valve surgery procedure as a safe alternative to a standard sternotomy, with reduced mortality rates, length of hospital stay, and reoperations for bleeding, along with a comparable risk of stroke.

There is a paucity of literature showing outcomes of MIMVS in older patients. Iribarne et al. demonstrated that it can be performed safely in patients at least 75 years old [19]. Furthermore, they noted that even though a minimally invasive approach was associated with slightly longer CPB and cross-clamp times than a conventional sternotomy, there were no significant differences regarding postoperative morbidity or mortality. Importantly, the mean and median durations of hospitalization were 3 and 1 day shorter, respectively, for patients who underwent an MIMVS procedure, findings with important implications for resource use. Our strategy consists of selecting MIMVR whenever possible even for elderly cases, thus we were not able to compare with a conventional sternotomy approach. In the present cohort, elderly patients 
( $\leq 80$ years old) who underwent MIMVR showed favorable rates for 5-year survival, freedom from a mitral valve-related reoperation, and freedom from cerebrovascular events and anticoagulation-related complications of $64 \%, 100 \%$, and $100 \%$, respectively. On the other hand, the cause of cardiac-related death could only be determined in 1 of 3 cases (septic shock after 2 months, fatal arrythmia after 3 months, unknown after 13 months). Nevertheless, the present results demonstrated the superiority of MIMVR in elderly patients the same as with the young group.

Factors with potential effects on the outcome of patients who undergo MIMVR are numerous and many confounding variables exist, though only dependent variables related to in-hospital mortality and prolonged hospital stay (>30 days) were examined in the present study. Univariate analysis indicated infectious endocarditis as a predictor, which might be related to the influence of post-operative antibiotic administration. Interestingly, previous cardiac surgery, concomitant operation, and elderly status were not significant. A multivariate logistic regression model of 409,904 valve procedures performed between 1994 and 2003 and cataloged in the Society of Thoracic Surgeons database demonstrated that the third most important preoperative variable influencing operative mortality is a reoperation (OR 1.61, $\mathrm{P}<0.001)$ [20]. However, the present data suggest that an RT approach after a previous median sternotomy is not an independent predictor of mortality $(P=0.26)$. This important finding indicates that this technique, in least in consideration of early outcome, should be the first choice for reoperative mitral valve surgery in patients who do not need concomitant aortic valve replacement or coronary revascularization. That is in concordance with previously reported data demonstrating equivalent or lower mortality rates, and less morbidity associated with an RT approach as compared to a reoperative sternotomy [16, 21, 22]. Nevertheless, findings regarding mid- and long-term outcomes are needed.

For treating mitral valve disease or degenerated bioprosthetic valve failure, transcatheter mitral valve replacement (TMVR) therapy has emerged as an encouraging option. Early reports have noted utilization of TAVR devices for degenerated surgical bioprosthetic valves (transcatheter mitral valve-in-valve implantation with balloon-expandable valves) (23) or annuloplasty rings (transcatheter mitral valve-inring implantation with balloon-expandable valves) (24), with reasonable results shown. Additionally, utilization of a TAVR valve in the mitral position has been described for treating patients with significant mitral annular calcification (MAC), though early results have demonstrated high rates of mortality and significant complications (25). Overall, survival (30-day all-cause mortality) after these various procedures has been greatest in patients who underwent a mitral valve-in-valve procedure $(6.2 \%)$, followed by valve-in-ring (9.9\%), with the worst results reported for valve-in-MAC (34.5\%) (26), and those findings raise questions regarding whether TMVR is an entirely appropriate alternative for surgical mitral valve surgery. Results presented thus far show that MIMVR is more effective. Additional long-term studies with larger numbers of enrolled patients will be necessary to better assess the efficacy of TMVR and MIMVR.

This study has some limitations. It was conducted in a retrospective manner and lacked a control group for appropriate comparisons. Thus, it was not possible to compare the present case series with a control group, since MIMVR has been our standard approach since 2014 and patients typically demand less 
invasive procedures. Even though this was a follow-up study conducted over 5 years, the number of patients was small and only 12 were found to be at risk at the 60-month follow-up examination. A welldesigned study with an appropriate sample size will be required in the future to validate the advantages of MIMVR.

To conclude, we found that MIMVR can be safely performed with encouraging short and mid-term outcomes, including elderly patients, with very low rates of conversion and mortality as compared to a conventional sternotomy procedure. In addition, a mitral valve re-operation can be safely and effectively performed through a smaller right thoracotomy. Finally, MIMVR was found useful for elderly patients. Based on these findings, we consider MIMVR to be an attractive alternative to conventional MVR.

\section{Abbreviations}

MIMVS: minimally invasive mitral valve surgery, MIMVR: minimally invasive mitral valve replacement, MVR: mitral valve replacement, RT: right mini-thoracotomy, IQR: interquartile range, CPB:

cardiopulmonary, AF: atrial fibrillation, TMVR: transcatheter mitral valve replacement, MAC: mitral annular calcification, COPD: chronic obstructive pulmonary disease, LVDd: left ventricular dimension diastole, LVDs: left ventricular dimension systole, LVEF: left ventricular ejection fraction, MVP: mitral valve plasty, OMC: open mitral commissurotomy, AVR: aortic valve replacement, TAR: total arch replacement, CABG: coronary artery bypass graft, MT: mini-thoracotomy, ST: median sternotomy, NA: not available, RFB: reoperation for bleeding, USA: United States of America, UK: United Kingdom

\section{Declarations}

\section{Acknowledgements}

Not applicable.

\section{Authors' contributions}

All authors contributed to the study design, to the analysis of the data, to writing the manuscript and in approving the final version.

\section{Funding}

The article has been created in the hospital setting without any type of financing.

\section{Availability of data and materials}

The datasets used and/or analysed during the current study are available from the corresponding author on reasonable request.

\section{Ethics approval and consent to participate}


This study was approved by the institutional ethical committee of Chiba-Nishi general hospital. All patients signed the informed consent for surgery. The sequential assignment patients signed the study consent form. The ethical committee advised that the study consent was not necessary in the historical cohort.

\section{Consent for publish}

Written informed consent to publish personal or clinical details were obtained from all the study participants.

\section{Competing interests}

The authors declare no competing interests.

\section{Author details}

Department of Cardiovascular Surgery, Chiba-Nishi General Hospital, Chiba, Japan

\section{References}

1. Nakayama T, Nakamura Y, Kanamori K, Hirano T, Kuroda M, Nishijima S, Ito Y, Tsuruta R, Hori T. Early and midterm results of minimally invasive aortic and mitral valve surgery via right mini-thoracotomy. J Card Surg. 2020;35(1):35-39.

2. Bowdish ME, D'Agostino RS, Thourani VH, Schwann TA, Krohn C, Desai N, Shahian DM, Fernandez FG, Badhwar V. STS Adult Cardiac Surgery Database: 2021 Update on Outcomes, Quality, and Research. Ann Thorac Surg 2021;111:1770-80

3. Botta L, Cannata A, Fratto P, Bruschi G, Trunflo S, Maneggia C, Martinelli L. The role of the minimally invasive beating heart technique in reoperative valve surgery. J Card Surg 2012;27:24-8.

4. Salhiyyah K, Taggart D. Beating-heart valve surgery: A systematic review. Asian Cardiovasc Thorac Ann 2009;17:650-8.

5. Nakayama T, Nakamura Y, Yasumoto Y, Yoshiyama D, Kuroda M, Nishijima S, Ito R, Tsuruta R, Hori T. Early and mid-term outcomes of minimally invasive mitral valve repair via right mini-thoracotomy: 5year experience with 129 consecutive patients. Gen Thorac Cardiovasc Surg. In press.

6. Modi P, Hassan A, Chitwood Jr. WR. Minimally invasive mitral valve surgery: a systematic review and meta-analysis. Eur J Cardiothorac Surg. 2008;34(5):943-52

7. Nakamura Y, Nishijima S, Kuroda M, Nakayama T, Tsuruta R, Yoshiyama D, Yasumoto $Y$, Ito $Y$. Perfusion strategy using axillary or femoral cannulation for minimally invasive cardiac surgery: experience in 270 patients with computed tomography-based criteria. Eur J Cardiothorac Surg. In press.

8. Modi P, Rodriguez E, Hargrove III WC, Hassan A, Szeto WY, Chitwood WR Jr. Minimally invasive videoassisted mitral valve surgery: A 12-year, 2-center experience in 1178 patients. J Thorac Cardiovasc 
Surg 2009;137(6):1481-7.

9. Glower DD, Siegel LC, Frischmeyer KJ, Galloway AC, Ribakove GH, Grossi EA, Robinson NB, Ryan WH, Colvin SB. Predictors of outcome in a multi- center port-access valve registry. Ann Thorac Surg 2000;70(September (3)):1054-9.

10. Casselman FP, VanSlycke S, Wellens F, DeGeest R, Degrieck I, Praet FV, Vermeulen Y, Vanermen $H$. Mitral valve surgery can now routinely be performed endoscopically. Circulation 2003;108(September (Suppl.1)):II48- II54.

11. Launcelott $S$, Ouzounian M, Buth KJ, Le' gare' J-F. Predicting in-hospital mortality after redo cardiac operations: development of a preoperative scorecard. Ann Thorac Surg 2012;94:778-84.

12. Byrne JG, Karavas AN, Adams DH, Aklog L, Aranki SF, Filsoufi F, Cohn LH. The preferred approach for mitral valve surgery after CABG: right thoracotomy, hypothermia and avoidance of LIMA-LAD graft. J Heart Valve Dis 2001;10(September (5)):584-90.

13. Ricci D, Pellegrini C, Aiello M, Alloni A, Cattadori B, D’Armini AM, Rinaldi M, Vigano M. Port-access surgery as elective approach for mitral valve operation in re-do procedures. Eur J Cardiothorac Surg 2010;37:920-5.

14. Sharony R, Grossi EA, Saunders PC, Schwartz CF, Ursomanno P, Ribakove GH, Galloway AC, Colvin SB. Minimally invasive reoperative isolated valve surgery: early and mid-term results. J Card Surg 2006;21:240-4.

15. Thompson MJ, Behranwala A, Campanella C, Walker WS, Cameron EWJ. Immediate and long- term results of mitral prosthetic replacement using a right thoracotomy beating heart technique. Eur $\mathrm{J}$ Cardiothorac Surg 2003;24:47-51; discussion 51.

16. Bolotin G, Kypson AP, Reade CC, Chu VF, Freund WL Jr, Nifong LW, Chitwood WR Jr. Should a videoassisted mini-thoracotomy be the approach of choice for reoperative mitral valve surgery? J Heart Valve Dis 2004;13:155-8; discussion 8.

17. Hiraoka A, Kuinose M, Totsugawa T, Chikazawa G, Yoshitaka H. Mitral valve reoperation under ventricular fibrillation through right mini-thoracotomy using three-dimensional videoscope. J Cardiothorac Surg 2013;8:81.

18. Losenno KL, Jones PM, Valdis M, Fox SA, Kiaii B, Chu MWA. Higher-risk mitral valve operations after previous sternotomy: endoscopic, minimally invasive approach improves patient outcomes. Can J Surg 2016;59:399-406.

19. Iribarne A, Easterwood R, Russo MJ, Chan EY, Smith CR, Argenziano M. Comparative effectiveness of minimally invasive versus traditional sternotomy mitral valve surgery in elderly patients. J Thorac Cardiovasc Surg. 2012;143:S86-90.

20. Rankin JS, Hammill BG, Ferguson TB Jr, Glower DD, O’Brien SM, DeLong ER, Peterson ED, Edwards $\mathrm{FH}$. Determinants of operative mortality in valvular heart surgery. J Thorac Cardiovasc Surg. 2006;131:547-57.

21. Burfeind WR, Glower DD, Davis RD, Landolfo KP, Lowe JE, Wolfe WG. Mitral surgery after prior cardiac operation: port-access versus sternotomy or thoracotomy. Ann Thorac Surg. 2002;74:S1323- 
5.

22. Sharony R, Grossi EA, Saunders PC, Schwartz CF, Ursomanno P, Ribakove GH, Galloway AC, Colvin SB. Minimally invasive reoperative isolated valve surgery: early and mid-term results. J Card Surg. 2006;21:240-4.

23. Gaia DF, Palma JH, deSouza JA, Ferreira CBND, Macedo MT, Gimenes MV, Buffolo E. Transapical mitral valve-in-valve implant: an alternative for high risk and multiple reoperative rheumatic patients. Int. J. Cardiol. 2012;154:e6-7

24. Wunderlich NC, Kische S, Ince H, Bozdag -Turanl. Transcatheter valve-in-ring implantation after a failed surgical mitral repair using a transseptal approach and a veno-arterial loop for valve placement. Catheter. Cardiovasc. Interv. 2014;84:1202-8

25. Guerrero M, Urena M, Pursnani A, Wang DD, Vahanian A, O'Neill W, Feldman T, Himbert D. Balloon expandable transcatheter heart valves for native mitral valve disease with severe mitral annular calcification. J. Cardiovasc. Surg. 2016;57:401-9

26. Yoon SH, Whisenant BK, Bleiziffer S, Delgado V, Dhoble A, et al. Outcomes of transcatheter mitral valve replacement for degenerated bioprostheses, failed annuloplasty rings, and mitral annular calcification. Eur. Heart J. 2019;40:441-51

\section{Figures}
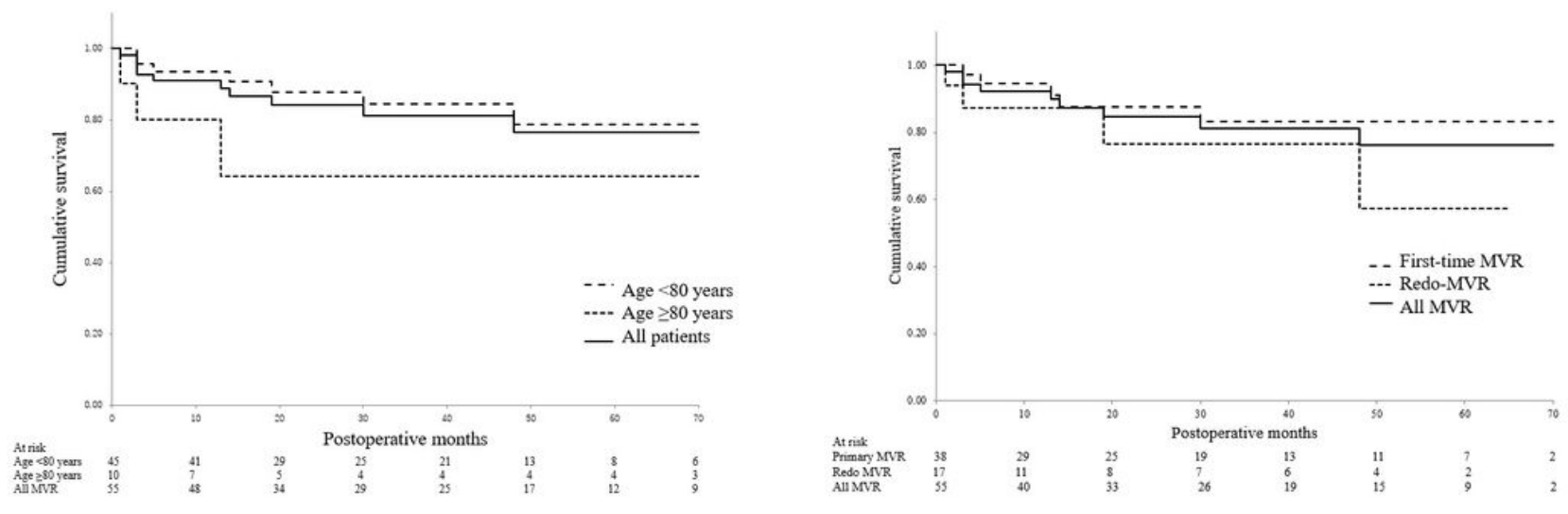

\section{Figure 1}

A. 1B. Freedom from late death for any reason. 

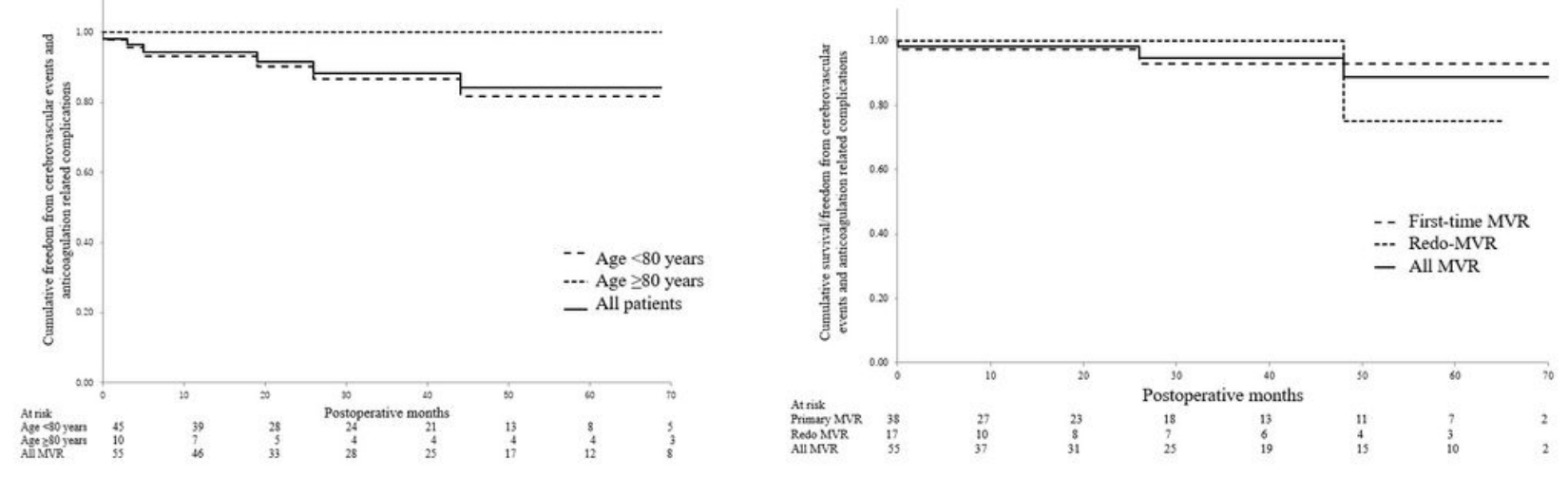

\section{Figure 2}

A. 2B. Freedom from cerebrovascular events and anticoagulation-related complications.

\section{Supplementary Files}

This is a list of supplementary files associated with this preprint. Click to download.

- JCTSMICSMVRtable2.pptx 